Manuscript ID ZUMJ-1909-1501 (R1)

DOI

10.21608/zumj.2019.16709.1501

ORIGINAL ARTICLE

\title{
CORRELATION OF CORONARY FLOW RESERVE, ANGIOGRAPHIC TIMI FLOW AND CONTRACTILE RESERVE IN PATIENTS WITH CARDIAC SYNDROME $X$
}

\author{
Ragab Abdel Salam ${ }^{1}$, Kamal Mahmoud ${ }^{2}$,Tamer Mohamed ${ }^{1}$, El-Sayed-Abdel-Kader ${ }^{3 r}$ \\ ${ }^{1}$ Cardiovascular Department, faculty of Medicine, Zagazig University, Zagazig, Egypt \\ ${ }^{2}$ Cardiovascular Department, Medical Research Institute, Alexandria University, \\ Alexandria, Egypt. \\ ${ }^{3}$ Cardiovascular Department, Alkappary Hospital, Specialized Medical Centers, Ministry of Health, \\ Alexandria, Egypt
}

\section{Corresponding Author: Elsayed Abdel Kader Elsayed \\ Cardiovascular \\ Department, Alkappary Hospital, Specialized Medical Centers, Ministry Of Health, Alexandria, Egypt E-mail: \\ Sayed345@yahoo.com}

Submit Date 2019-09-10 Revise Date 2019-09-22 Accept Date 2019-09-24

\begin{abstract}
Background: The cardiovascular syndrome $\mathrm{X}$ includes chest pain with positive stress test, and coronary arteries that appear normal in angiography with possible extra cardiac causes of chest pain, ruled out. The aim of the study is to evaluate the correlation between TIMI flow, contractile reserve and the coronary flow reserve in patients with cardiac syndrome $\mathrm{X}$ using TTDE. Methods: 60 patients with cardiac syndrome X, and 30 individuals as a control. All studied individuals were subjected to full history taking, full clinical examination, routine laboratory examination, resting ECG, treadmill stress ECG, Dobutamine stress echocardiogram and Transthoracic Doppler Echocardiogram .Results: all individuals were referred to TTDE and DSE in cardiac unit of Zagazig University Hospital from January 2017 to March 2019. The patients group showed a significant decrease in contractile reserve, a highly significant lower value of a percentage change of MAPSE with $p$ value $<0.007$. The decrease of CFVR was associated with an increase of exercise time, ST segment depression and Postrecovery time. The decrease of CFVR was associated with the decrease of MAPSE during maximum dose of dobutamine. With a highly significant relationship between the decrease CFVR and the percentage decrease of MAPSE. CONCLUSION: The impaired coronary microvascular function could be correlated with a MAPSE reserve as a contractile reserve marker for CMVD and a highly positive results of exercise ECG.
\end{abstract}

Keywords:

Cardiac syndrome X - coronary flow reserve - contractile reserve-Dobutamine stress echocardiogram.

\section{INTRODUCTION}

$\mathrm{C}$ ardiovascular disease (CVD) is the leading cause of death worldwide and coronary artery disease (CAD) is the most common type of CVD. Up to $20-30 \%$ of patients presenting with chest discomfort characteristic of angina demonstrate no signs of obstructive CAD upon angiography. CSX can be defined as exercise induced angina like chest discomfort, ST segment depression during angina and normal epicardial coronary artery cardiac catheterization $(1,2,3,4,5) \cdot$ With no spontaneous or inducible epicardial coronary spasm and absence of systemic diseases associated with microvascular dysfunction (MVD) as hypertrophic cardiomyopathy (HCM) or diabetes(6). The diagnosis of CSX requires history of exercise induced chest pain with ST segment depression induced by exercise electrocardiogram (ECG) testing with no obstructive CAD on angiography. Many patients with CSX do not complete the exercise test because of fatigue and mild chest 
discomfort. Left ventricular (LV) function is usually normal at rest and during stress, unlike the situation in obstructive CAD, in which the function often become impaired during stress. Microvascular function is evaluated by testing vascular flow response to vasodilator stimuli with various techniques and calculating CFR.CFR is a ratio between hyperemic and basal CBF. The most widely used substances are adenosine and dipyridamole for endothelium independent vasodilatation. While the acetylcholine and cold pressure for endothelium dependent vasodilatation. A CFR $<2.5$ is usually considered as diagnostic for CMD although a CFR of $<2$ might be more specific and diagnostic. However exclusion of significant vasoconstriction of epicardial coronaries and microvascular spasm are needed for accuracy (7). Coronary microvascular dysfunction affects the myocardial contractility in women with angina and non-obstructive CAD. Which concluded that the global longitudinal strain (GLS) reserve was significantly lower in women with CMVD this suggests that the CMVD contribute to ischemia with inability of left ventricular function to increase during hyperemic stress in women with angina \& non obstructive CAD. Based on the new emerging marker for contractile reserve namely percentage change of MAPSE, a noninvasive evaluation of contractile reserve similar to percentage change of GLS as the both reflect the longitudinal fiber shortening change under stress condition.

\section{Methods:}

60 patients with normal coronary anatomy were selected according to inclusion and exclusion criteria as a patients group with cardiac syndrome $X$, in addition to 30 individuals as a control group with negative exercise stress ECG and not referred to coronary angiography. Written informed consent was obtained from all participants and the study was approved by the research ethical committee of Faculty of Medicine, Zagazig University. The work has been carried out in accordance with The Code of Ethics of the World Medical Association (Declaration of Helsinki) for studies involving humans.

Inclusion criteria: All patients presenting with angina like chest pain of low to intermediate probability of coronary artery disease (CAD).

Exclusion criteria: Patients with

Confirmed ischemic heart diseases (stable, unstable angina, myocardial infarction).

Previous history of percutaneous coronary intervention (PCI) or previous coronary artery bypass grafting $\mathrm{CABG}$.

Congenital heart diseases.

Cardiac muscle diseases (cardiomyopathies).

Hypertensive patients with or without left ventricular hypertrophy.

Valvular heart diseases.

Diabetes mellitus, dyslipidemia.

Hepatic or renal impairment.

All studied individuals (patients \& control groups) were subjected to

Full history taking.

Complete clinical examination

Routine laboratory tests.

12-leads resting ECG.

Treadmill stress ECG: (exercise time in minutes, ST segment depression in $\mathrm{mm}$, postrecovery time in minute).

Coronary angiography: myocardial blood flow is assessed angiographically using the thrombolysis in myocardial infarction (TIMI) score for qualitative grading of coronary flow. Evaluation of contractile reserve using Dobutamine stress echocardiogram: using the new emerging marker of change in longitudinal fiber shortening during stress with dobutamine and the mitral annular plane systolic excursion, (MAPSE \% change). MAPSE is measured at basal echo study both at septal and free wall MVLs and mean is obtained, again it is measured at maximal dose of dobutamine in apical 44 chamber view and percentage change is calculated by subtracting MAPSE at maximal dose from baseline one divided by baseline one

Transthoracic Doppler Echocardiogram (TTDE): for exclusion of structural, valvular and congenital heart diseases and for assessment of coronary flow velocity reserve (CFVR).

CFVR is assessed using (Healthcare GE vivid S5) with 3.5 -5 MHZ transducer:-

1- The acoustic window was around the mid calvicular line in the fourth and fifth 
intercostal space in the left lateral decubitus position.

2- The color Doppler flow mapping gain was adjusted to provide the optimal imaging.

3- Firstly the left ventricle was imaged in the long axis cross -section and the ultrasound beam was inclined laterally with slight counter clockwise and superior angulation.

4- Next, the coronary blood flow in the distal portion of left anterior descending artery was visualized by $5 \mathrm{MHZ}$ color Doppler.

5- The coronary blood flow velocity was measured with pulsed Doppler, as the sample volume was placed on the color signal in distal LAD.

The average diastolic peak velocity was measured;-

(a) First at the baseline.

(b) During adenosine infusion of $140 \mathrm{ug} / \mathrm{kg}$ body weight per min over 90 seconds in an anticubital vein to induce hyperemia

6- Coronary Flow Reserve (CFR) was calculated as the ratio of peak hyperemic to basal peak diastolic velocity.

\section{Statistical analysis}

Data were fed to the computer and analyzed using IBM SPSS (Statistical Package for the Social Sciences) software package version 20.0 (Armonk, NY: IBM Corp).

Qualitative data were described using number and percent. The KolmogorovSmirnov test was used to verify the normality of distribution.

Quantitative data were described using range (minimum and maximum), mean, standard deviation and median. Significant of the obtained results was judged at the 5\% level.

The used tests were

1- Chi-square test: For categorical variables, to compare between different groups.

2- Fisher, s Exact or Monte Carlo correction: Correction for chi-square when more than $20 \%$ of the cells have expected count less than 5 .

3- Student t-test: For normally distributed quantitative variables, to compare between studied groups.

4- Pearson coefficient: To correlate between two normally distributed quantitative variables.

5- Mann Whitney test: For abnormally distributed quantitative variables, to compare between two studied groups.

\section{RESULTS}

This a study included a group of 60 patients with cardiac syndrome $\mathrm{X}$ and a control group of 30 individuals. Both groups were referred to TTDE and DSE in cardiac unit of Zagazig University Hospital from January 2017 to March 2019.

\section{Prevalence of risk factors for CAD between studied groups:-}

Patients group included 20 males $(33.3 \%)$ and 40 females $(66.7 \%)$, while the controls group included 12 males (40\%) and 18 females $(60 \%)$ with no significant differences between both groups. The patients group included 15 smokers (25\%of patients) and controls group included 8 smokers (26.7\% of controls) with no significant difference between both groups.19 patients (31.7\%) had a family history of IHD and 12 controls had a family history of IHD (40\%) with no significant difference between both studied groups. The age of the patients ranged between 46 and 70 years while the controls ranged between 30 and 73 years with no significant difference between both studied groups.

Table (1): At baseline there was no significant changes between both groups, but the patients group had a highly significant lower value of MAPSE at maximal dose of dobutamine ( $\mathrm{p}$ value <0.007) and as a percentage change of MAPSE with $\mathrm{p}$ value $<0.007$. Thus the contractile reserve is highly significantly lower in patients group as reflected by MAPSE. Table (2) showed significant decrease in CFVR in patient group

Correlation between the CFVR and the contractile reserve as reflected from dobutamine stress echocardiography. Showed a significant relatioship between the decrease of CFVR and the decrease of mitral annular plane systolic excursion (MAPSE) during maximum dose of dobutamine (directly proportional relation). With a highly significant relationship between the decrease CFVR and the percentage decrease of MAPSE (=difference between MAPSE at 
baseline and at high dose of dobutamine (baseline MAPSE).

Table (3): showed Correlation between CFVR with different parameters in Patient Table (4): Parameters affecting the CFVR: the most independed significant variables were the BMI and the extent of ST segment depression.

Table (5): Agreement (sensitivity, specificity) for post recovery time to predict CFVR $\geq 2$ from $\mathrm{CFVR}<\mathbf{2}$

Table (6): Distribution of the studied cases according to exercise ECG data in patient group Table (7): Relation between CFVR and different parameters in Patient group

Table (1): Comparison between the two studied groups according to MAPSE

\begin{tabular}{|c|c|c|c|c|c|c|}
\hline MAPSE & \multicolumn{2}{|c|}{$\begin{array}{l}\text { Patient } \\
(n=60)\end{array}$} & \multicolumn{2}{|c|}{$\begin{array}{l}\text { Control } \\
(\mathrm{n}=30)\end{array}$} & $\begin{array}{l}\text { Test } \\
\text { Sig. }\end{array}$ & p \\
\hline \multicolumn{7}{|l|}{ At baseline } \\
\hline Min - Max & \multicolumn{2}{|c|}{$1.40-2.50$} & \multicolumn{2}{|c|}{$1.60-2.40$} & \multirow{3}{*}{$\begin{array}{l}\mathrm{t}= \\
1.291\end{array}$} & \multirow[t]{3}{*}{0.200} \\
\hline Mean \pm SD & \multicolumn{2}{|c|}{$2.03 \pm 0.24$} & \multicolumn{2}{|c|}{$1.96 \pm 0.21$} & & \\
\hline Median & \multicolumn{2}{|l|}{2.0} & \multicolumn{2}{|c|}{1.95} & & \\
\hline At maxi dose & No. & $\%$ & No. & $\%$ & \multirow{3}{*}{$\begin{array}{l}\chi^{2}= \\
24.202^{*}\end{array}$} & \\
\hline$\leq 2$ & 48 & 80.0 & 8 & 26.7 & & \multirow[t]{2}{*}{$<0.001^{*}$} \\
\hline$>2$ & 12 & 20.0 & 22 & 73.3 & & \\
\hline Min - Max & \multicolumn{2}{|c|}{$1.15-2.40$} & \multicolumn{2}{|c|}{$1.80-2.70$} & \multirow{3}{*}{$\begin{array}{l}\mathrm{t}= \\
6.255^{*}\end{array}$} & \multirow[t]{3}{*}{$<0.001^{*}$} \\
\hline Mean \pm SD & \multicolumn{2}{|c|}{$1.85 \pm 0.28$} & \multicolumn{2}{|c|}{$2.22 \pm 0.23$} & & \\
\hline Median & \multicolumn{2}{|c|}{1.83} & \multicolumn{2}{|c|}{2.27} & & \\
\hline \multicolumn{7}{|l|}{ A\% MAPSE } \\
\hline Min - Max & \multicolumn{2}{|c|}{$-26.30-75.0$} & \multicolumn{2}{|c|}{$0.50-33.30$} & \multirow{3}{*}{$\begin{array}{l}\mathrm{U}= \\
30.000\end{array}$} & \multirow[t]{3}{*}{$<0.001^{*}$} \\
\hline Mean \pm SD & \multicolumn{2}{|c|}{$-7.45 \pm 12.35$} & \multicolumn{2}{|c|}{$12.99 \pm 7.84$} & & \\
\hline Median & \multicolumn{2}{|c|}{-5.15} & \multicolumn{2}{|c|}{11.0} & & \\
\hline
\end{tabular}

$\chi^{2}$ : Chi square test $t$ : Student t-test

U: Mann Whitney test

$\mathrm{p}$ : $\mathrm{p}$ value for comparison between the two studied groups

*: Statistically significant at $\mathrm{p} \leq 0.05$

Table (2): Comparison between the two studied groups according to coronary flow velocity reserve

\begin{tabular}{|c|c|c|c|c|c|c|c|}
\hline \multicolumn{2}{|c|}{ Coronary flow velocity reserve $(\mathrm{cm})$} & \multicolumn{2}{|c|}{$\begin{array}{l}\text { Patient } \\
(n=60)\end{array}$} & \multicolumn{2}{|c|}{$\begin{array}{l}\text { Control } \\
(n=30)\end{array}$} & \multicolumn{2}{|c|}{$\begin{array}{l}\text { Test of p } \\
\text { Sig. }\end{array}$} \\
\hline & Basal & \multirow{2}{*}{\multicolumn{2}{|c|}{$21.0-46.0$}} & \multirow{2}{*}{\multicolumn{2}{|c|}{$19.0-33.0$}} & & \\
\hline & Min - Max & & & & & \multirow{3}{*}{$\begin{array}{l}t= \\
8.271^{*}\end{array}$} & \multirow[t]{3}{*}{$<0.001^{*}$} \\
\hline & Mean $\pm \mathrm{SD}$ & \multicolumn{2}{|c|}{$34.38 \pm 7.54$} & \multicolumn{2}{|c|}{$24.27 \pm 4.06$} & & \\
\hline & Median & \multicolumn{2}{|c|}{36.0} & \multicolumn{2}{|c|}{22.50} & & \\
\hline & Hyperemic & \multirow{2}{*}{\multicolumn{2}{|c|}{$35.0-442.0$}} & \multirow{2}{*}{\multicolumn{2}{|c|}{$54.0-98.0$}} & \multirow{4}{*}{$\begin{array}{l}U= \\
687.00\end{array}$} & \multirow{4}{*}{0.068} \\
\hline & Min - Max & & & & & & \\
\hline$\vec{z}$ & Mean \pm SD & \multicolumn{2}{|c|}{$68.43 \pm 51.85$} & \multicolumn{2}{|c|}{$71.20 \pm 12.18$} & & \\
\hline 皆 & Median & \multicolumn{2}{|c|}{66.50} & \multicolumn{2}{|c|}{69.50} & & \\
\hline \multicolumn{8}{|l|}{ CFVR } \\
\hline $1.5-<2$ & & 47 & 78.3 & 0 & 0.0 & \multirow{2}{*}{$\begin{array}{l}\chi^{2}= \\
49.186^{*}\end{array}$} & \multirow[t]{2}{*}{$<0.001^{*}$} \\
\hline$\geq 2$ & & 13 & 21.7 & 30 & 100.0 & & \\
\hline Min - Max & & \multicolumn{2}{|c|}{$1.35-2.40$} & \multicolumn{2}{|c|}{$2.57-3.70$} & \multirow{3}{*}{$\begin{array}{l}\mathrm{t}= \\
20.846^{*}\end{array}$} & \multirow[t]{3}{*}{$<0.001^{*}$} \\
\hline Mean \pm SD & & \multicolumn{2}{|c|}{$1.78 \pm 0.25$} & \multicolumn{2}{|c|}{$2.94 \pm 0.25$} & & \\
\hline Median & & \multicolumn{2}{|c|}{1.76} & \multicolumn{2}{|c|}{2.93} & & \\
\hline
\end{tabular}

$\chi^{2}$ : Chi square test t: Student t-test

$\mathrm{p}$ : $\mathrm{p}$ value for comparison between the two studied groups

*: Statistically significant at $\mathrm{p} \leq 0.05$ 
Table (3): Correlation between CFVR with different parameters in Patient groups $(n=60)$

\begin{tabular}{|l|l|l|}
\hline & CFVR & \\
\hline Exercise time & $\mathbf{r}$ & $\mathbf{p}$ \\
\hline ST segment & $0.446^{*}$ & $<0.001^{*}$ \\
\hline Post recovery time & $-0.549^{*}$ & $<0.001^{*}$ \\
\hline
\end{tabular}

r: Pearson coefficient

*: Statistically significant at $\mathrm{p} \leq 0.05$

Table (4): Multivariate analysis for the parameters affecting CFVR $(n=60)$

\begin{tabular}{|l|l|l|l|l|} 
& \multicolumn{2}{l}{ Multivariate } & 95\% CI. & p \\
& & B & LL & \\
\hline FH of IHD & 0.014 & -0.176 & 0.205 & 0.881 \\
\hline TG & 0.000 & -0.004 & 0.003 & 0.914 \\
\hline BMI & -0.058 & -0.093 & -0.023 & $0.002^{*}$ \\
\hline Exercise time & -0.030 & -0.083 & 0.023 & 0.267 \\
\hline ST segment & -0.309 & -0.607 & -0.011 & $0.042^{*}$ \\
\hline A\% MAPSE & 0.000 & -0.005 & 0.005 & 0.885 \\
\hline
\end{tabular}

B: Unstandardized Coefficients

CI: Confidence interval

LL: Lower limit

UL: Upper Limit

*: Statistically significant at $\mathrm{p} \leq 0.05$

Table (5):Agreement (sensitivity, specificity) for post recovery time to predict CFVR $\geq 2$ from CFVR $<2$

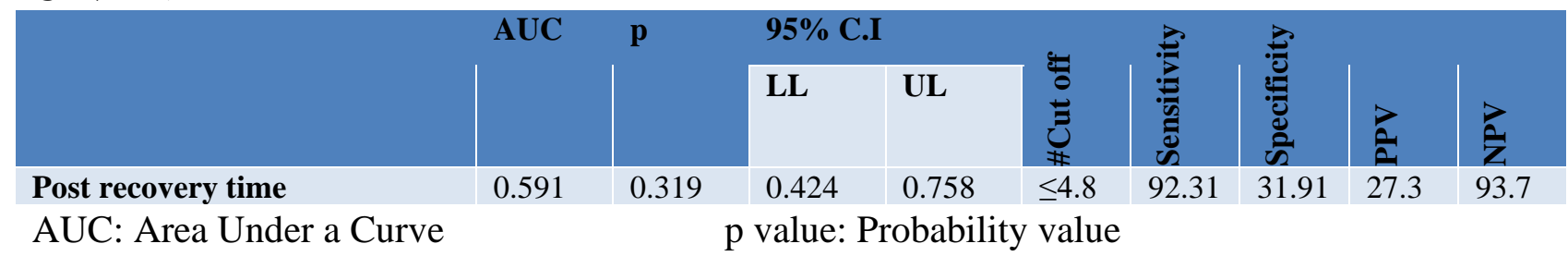

CI: Confidence Intervals

NPV: Negative predictive value PPV: Positive predictive value

*: Statistically significant at $\mathrm{p} \leq 0.05$

\#Cut off was choosed according to Youden index

Table (6): Distribution of the studied cases according to exercise ECG data in patient group

\begin{tabular}{|c|c|c|}
\hline \multirow{2}{*}{$\begin{array}{l}\text { Exercise ECG data } \\
\text { Exercise time }\end{array}$} & \multirow[t]{2}{*}{ No. } & $\%$ \\
\hline & & \\
\hline$>6$ & 48 & 80.0 \\
\hline$\leq 6$ & 12 & 20.0 \\
\hline Min - Max & \multicolumn{2}{|c|}{$5.0-12.0$} \\
\hline Mean \pm SD & \multicolumn{2}{|c|}{$8.36 \pm 2.02$} \\
\hline Median & \multicolumn{2}{|c|}{8.90} \\
\hline \multicolumn{3}{|l|}{ ST segment } \\
\hline$<1.5$ & 31 & 51.7 \\
\hline$\geq 1.5$ & 29 & 48.3 \\
\hline Min - Max & \multicolumn{2}{|c|}{$1.0-2.0$} \\
\hline Mean \pm SD & \multicolumn{2}{|c|}{$1.44 \pm 0.35$} \\
\hline Median & \multicolumn{2}{|c|}{1.40} \\
\hline \multicolumn{3}{|l|}{ Post recovery time } \\
\hline$<4$ & 35 & 58.3 \\
\hline$\geq 4$ & 25 & 41.7 \\
\hline Min - Max & \multicolumn{2}{|c|}{$1.60-5.30$} \\
\hline Mean \pm SD. & \multicolumn{2}{|c|}{$3.25 \pm 1.46$} \\
\hline Median & \multicolumn{2}{|c|}{2.25} \\
\hline
\end{tabular}


Table (7): Relation between CFVR and different parameters in Patient group

\begin{tabular}{|c|c|c|c|c|c|c|}
\hline & \multirow[t]{2}{*}{$\mathbf{N}$} & \multicolumn{3}{|l|}{ CFVR } & \multirow[t]{2}{*}{ t } & \multirow[t]{2}{*}{ p } \\
\hline & & Min. - Max. & Mean \pm SD & Median & & \\
\hline \multicolumn{7}{|l|}{ Sex } \\
\hline Male & 20 & $1.43-2.37$ & $1.74 \pm 0.22$ & 1.72 & \multirow[t]{2}{*}{1.027} & \multirow[t]{2}{*}{0.309} \\
\hline Female & 40 & $1.35-2.40$ & $1.81 \pm 0.26$ & 1.76 & & \\
\hline \multicolumn{7}{|l|}{ Smoking } \\
\hline Negative & 45 & $1.35-2.40$ & $1.81 \pm 0.26$ & 1.78 & \multirow[t]{2}{*}{1.357} & \multirow[t]{2}{*}{0.180} \\
\hline Positive & 15 & $1.78-2.37$ & $1.71 \pm 0.21$ & 1.71 & & \\
\hline \multicolumn{7}{|c|}{ FH of IHD } \\
\hline Negative & 41 & $1.52-2.40$ & $1.86 \pm 0.22$ & 1.80 & \multirow[t]{2}{*}{$4.173^{*}$} & \multirow[t]{2}{*}{$<0.001^{*}$} \\
\hline Positive & 19 & $1.35-2.37$ & $1.61 \pm 0.22$ & 1.55 & & \\
\hline
\end{tabular}

\section{t: Student t-test}

$\mathrm{p}$ : $\mathrm{p}$ value for associated between CFVR and different parameters

$*$ : Statistically significant at $\mathrm{p} \leq 0.05$

\section{DISCUSSION}

Historically CSX was considered to be a benign condition, but recent reports have showed that CSX patients have a higher prevalence of adverse cardiovascular events and a poor quality of life when compared to normal individuals.

This study aimed to investigate the relatioship between coronary flow velocity reserves (CFVR), contractile reserve and other risk factors of ischemic heart disease (IHD). Transthoracic Doppler echocardiography (TTDE) as a noninvasive tool for evaluation of cardiac structure and function can also be used for assessment of CFR in a noninvasive feasible, reliable and reproducible fashion following a standardized protocol with different vasodilator stimuli (8).

Luca Lanzarini et al (9) studied the results of dobutamine stress echocardiography (DSE) in ten patients' with CSX. All patients underwent DSE after interruption of any antianginal therapy There was an increase in EF reserve and a decrease in MAPSE reserve, while the EF represents the global results of both longitudinal and circumferential contraction, the MAPSE represents the longitudinal contraction, so with the normal $\mathrm{EF}$, the compensatory increased circumferential deformation might mask the reduced longitudinal deformation. Reduced long axis deformation results from dysfunctional or stressed longitudinal myofibers due to endocardial or epicardial ischemia, fibrosis or increased wall stress. Moreover, reduced MAPSE could be used as a sensitive early marker of LV systolic dysfunction (10).

Willenheimer et al, (11) demonstrated that MASPE was reduced in 88 out of 1350 consecutive patients with visual evaluated normal LV regional wall motion and these patients with reduced MAPSE had either prior myocardial infarction $(60 \%)$, or coronary artery disease without infarction $(33 \%)$, or uncontrolled hypertension (2\%) while definitive evidence for CVD was absent in only $4 \%$ patients with reduced MAPSE. This suggests that decrease MAPSE, in the case of normal LV regional wall motion could serve as an echocardiographic functional sign for myocardial abnormalities predominantly indicating subendocardial dysfunction or CMVD.

This study showed that CSX patients with reduced CFVR had a highly significant relatioship with qualitative risk factor family history of IHD and quantitative risk factors of IHD SBP, DBP, total cholesterol LDL-C, TG a ratio of TG/HDL, BMI and glycemic level, there were a negative correlation to CFVR ( inversely proportional to CFVR). And significant relation with $\mathrm{HR}$ that was inversely proportional to CFVR with a significant positive correlation to HDL-C, the lower value of HDL-C with lower CFVR.

Bajaj NS, et al (12) followed consecutive patients undergoing evaluation for CAD with cardiac stress posterion emission tomography (PET) demonstrating normal perfusion $\mathrm{N}=828$. The results showed an inverted independed J-shaped relationship was observed between BMI and CFR. The authors concluded that CMVD was 
independedly associated with elevated BMI and adverse outcome and was a discriminator of risk than BMI and tradional risk factors. The study agreed with our study the BMI is independed variable for a decrease CFVR and so associated with CMVD.

According to our study, the receiver operating characteristic (ROC) curve showed the predictive power of CFVR (CMVD) by postrecovery time with cutoff point of $\leq 4.8$ as a discriminative power distinguishing CFVR $<2$ from CFVR $\geq 2$ with a sensitivity value of $92.31 \%$ and negative predictive value(NPV) $93.7 \%$, while specificity was $31.91 \%$ and positive predictive value(PPV) $27.3 \%$ with AUC 0.591 .

Contractile reserve as reflected by MAPSE reserve could predict the CFVR as discriminative power distinguishing CFVR $<2$ from CFVR $>/=2$ with significant value $\mathrm{P}<0.05$, sensitivity $84.62 \%$, NPV $0.93 .3 \%$, specificity $59.57 \%$ \& PPV $36.7 \%$ and UAC 0.695 .

Erkan Yildirim, et al (13) evaluated the coronary flow by the coronary clearance time in patients with CSX. Thrombosis in myocardial infarction frame count (TFC) and coronary clearance frame count (CCFC) were calculated offline. A total of 71 patients with CSX and 61 control patients were included in the study. The TFC of LAD, CFX and RCA were similar between the two groups while the mean CCFC were significantly longer in CSX group compared with the control group. The study concluded that CCFC is a simple quantitative and highly reproducible method as a marker of CMVD.

\section{Limitations:}

Limited number of male patients.

Patients with previous history of CABG and have angina-like chest pain with normal coronary angiogram not included in our study.

Patients with previous history of PCI and angina-like chest pain with normal coronary angiogram not included in our study. Invasive validated marker for prediction of CMVD and correlation with noninvasive markers such as coronary clearance frame count not included in our study.

Most of Significant variables for detection of CFVR were univariate.
Loss of follow up of the Patients with highly significant and significant variables with CFVR impairment (CMVD).

\section{CONCLUSION}

The impairment of coronary microvascular function could be correlated with a MAPSE reserve as a contractile reserve marker for CMVD and a highly positive results of ETT in form of postrecovery time, extent of ST segment depression \& exercise capacity.

\section{RECOMMENDATION}

- TTDE is a good noninvasive tool for assessment of CFVR even with those having poor windows endocardial contrast echocardiography can be utilized.

- A noninvasive echocardiographic marker MAPSE need extended and future trials to confirm its prediction value in assessment of CMVD.

- Validated scoring system for discrimination between CSX patients and obstructive CAD patients is needed for cost benefit which ones need an invasive evaluation and for close observation for those with high risk score.

- Investigation of new invasive markers may be needed for correlation with noninvasive markers to get a good, feasible, practicable and highly validated noninvasive markers such as coronary clearance frame count (CCFC), coronary sinus flow time (CSFT).

- Longer follow up period is required for highly risk CSX patients.

Declaration of interest:

The authors report no conflicts of interest.

The authors alone are responsible for the content and writing of the paper.

Funding information

None declared.

\section{REFERENCE}

1. Kaski JC, Rosano GM, Collins $P$, Nihoyannopoulos P, Maseri A, PooleWilson PA. Cardiac syndrome X: clinical characteristics and left ventricular function. Long-term follow-up study. J Am Coll Cardiol. 1995; 25(4):807-814.

2. Richard N. Fogoros MD. Microvascular angina or cardiac syndromeX.2018.Availablefrom: verywellhealth. Accessed in: Feb, 2019.

3. Cannon RO, Epstein SE. Microvascular angina as a cause of chest pain with angiographically normal coronary arteries. Am J Cardiol. 1988; 61:1338-1343.Wachira JK et 
al., 2013

4. Melikian N, De Bruyne B, Fearon WF, MacCarthy PA. The pathophysiology and clinical course of the normal coronary angina syndrome (cardiac syndrome $\mathrm{X}$ ). Prog Cardiovasc Dis. 2008; 50(4):294-310.

5. Parsyan A, Pilote L. Cardiac syndrome X: mystery continues. Can J Cardiol. 2012; 28(Supple 2):S3-S6.

6. Lanza GA, Camici PG, Galiuto L, Niccoli G, Pizzi C, Di Monaco A et al, Methods to investigate coronary microvascular function in clinical practice" On behalf of Gruppo di Studio di Fisiopatologia Coronarica e Microcircolazione, Società Italiana di cardiologia. J Cardiovasc Med (Hagerstown). 2013; 14:1-18.

7. Sucato V, Evola S, Novo G, Bronte E. Stable microvascular angina :instrumental evaluation of coronary microvascular dysfunction with coronary angiography and myocardial scintigraphy. Int J Cardiol. 2014; 171: e127e128.

8. Simova I. Coronary Flow Velocity Reserve Assessment with Transthoracic Doppler Echocardiography. Eur Cardiol Rev. 2015; 10(1): 12 .

9. Lanzarini L, Previtali M, Fetiveau R, Poli A.Results of dobutamine stress echocardiography in patients with cardiac syndrome X. Int J Card imaging. 1994; 10:145-148.

10.Hu K, Liu D, Herrmann S, Niemann M, Gaudron PD, Voelker W et al, Clinical implication of Mitral Annular Plane Systolic Excursion (MAPSE) for patients with cardiovascular disease. Eur Heart J Cardiovasc Imaging. 2013; 14(3):205-212.

11.Willenheimer R, Rydberg E, Stagmo M, Gudmundsson P, Ericsson G, Erhardt L ,et al. Echocardiographic assessment of left atrioventricular plane displacement as a complement of left ventricular regional wall motion evaluation in detection of myocardial dysfunction. Int J Cardiovasc Imaging. 2002; 18:181-186.

12. Bajaj NS, Osborne MT, Gupta A, Tavakkoli A, Bravo PE, Vita T, et al. Coronary microvascular dysfunction and coronary flow reserve in obese patients. Am Coll Cardiol. 2018; 72:707-717.

13. Erkan Yildirim, Uygar Cagdas Yuksel, Murat Celik, Baris Bugan, Mutlu Gungor, Yalcin Gokoglan,et al. Evaluation of coronary flow by the coronary clearance time in patients with cardiac syndrome X. J Int Med Res. 2018; 46(3):1121-1129.

\section{How to Cite}

Elsayed, E., Mahfouz, R., mahmoud, K., Mostafa, T. Correlation of the coronary flow reserve, the angiographic TIMI flow and the contractile reserve in patients with cardiac syndrome $\mathrm{x}$. Zagazig University Medical Journal, 2021; (924-931): -. doi: 10.21608/zumj.2019.16709.1501 